\title{
Characterization and Molecular Mapping of Two Novel Genes Resistant to Pythium Stalk Rot in Maize
}

\author{
Canxing Duan, ${ }^{1}$ Fengjing Song, ${ }^{1}$ Suli Sun, ${ }^{1}$ Cheng Guo, ${ }^{2}$ Zhendong Zhu, ${ }^{1}$ and Xiaoming Wang $1, \dagger$ \\ ${ }^{1}$ Institute of Crop Sciences, Chinese Academy of Agricultural Sciences/National Key Facility for Crop Gene Resources and Genetic \\ Improvement, Beijing 100081, China; and 2Institute of Plant Protection, Gansu Academy of Agricultural Sciences, Lanzhou 730070, China \\ Accepted for publication 12 October 2018.
}

ABSTRACT

\begin{abstract}
Pythium stalk rot caused by Pythium inflatum is becoming a more and more serious disease in maize, and it has caused severe yield loss in China in recent years. Deployment of resistant maize varieties is the most effective way to control this disease. Searching for the resistant maize germplasm and identifying the resistance genes are the vital processes in the breeding program. The maize inbred line X178 previously showed high resistance to Pythium stalk rot. Thus, this study aimed to reveal the gene(s) resistance to Pythium stalk rot in X178 by resistance inheritance analysis using the derived $F_{2}$ and $F_{2: 3}$ genetic populations. The results showed that two independently inherited dominant genes, designated RpiX178-1 and RpiX178-2, carried by X178 are responsible for its resistance relative to the susceptible parent Ye107; they are located on
\end{abstract}

regions of maize chromosome (chr.) 1 bin 1.09 and chr. 4 bin 4.08, respectively, and flanked by markers umc2047 and bnlg1671 as well as bnlg1444 and umc1313, respectively, by linkage analysis. Subsequently, RpiX178-1 was finely mapped between markers SSRZ8 and IDP2347, with genetic distances of 0.6 and $1.1 \mathrm{cM}$, respectively, and the physical distance of the target region was about $700 \mathrm{~kb}$. RpiX178-2 was also further located between markers bnlg1444 and umc2041, with a genetic distance of $2.4 \mathrm{cM}$. Moreover, we confirmed that the two genes RpiX1781 and RpiX178-2 were newly identified and different from those genes known on chrs. 1 and 4 according to results of allelism testing. Herein, we newly identified two genes resistant to $P$. inflatum, which provided important genetic information for resistance to Pythium stalk rot in maize.
Maize is a major food and feed crop, because it is a high-energy crop, and it is also utilized as an industrial raw material in China. Therefore, it plays an important role in the national economy and agriculture production (Duan et al. 2012). Since 2003, the acreage dedicated to maize cultivation in China has been continuously increasing, with maize being the most highly cultivated crop in China. Therefore, reliable maize production is necessary to maintain China's food security and agricultural stability (Duan et al. 2015b). Pythium stalk rot, which is caused by Pythium inflatum, is a soilborne disease that severely affects maize production. This disease can occur in diverse environments, but it is more prevalent with excessive humidity, and it can strikingly affect susceptible maize inbred lines and varieties, resulting in severe yield and quality reductions (Duan et al. 2015b; Khokhar et al. 2014). In recent years, changes in climatic conditions, cultivation methods, and the maize varieties utilized have contributed to Pythium stalk rot becoming a major disease in the Huang-Huai region and northwestern China. Efforts to control the disease via chemical, agricultural, or biological means have had little success. Therefore, selective breeding and the development of disease-resistant varieties are the most economical, effective, and sustainable measures for controlling Pythium stalk rot.

Successfully breeding resistant varieties involves screening for resistant resources, performing genetic studies relating to resistance, and mining and mapping resistance genes. Previous

${ }^{\dagger}$ Corresponding author: X. Wang; wangxiaoming@caas.cn

Funding: This project was supported by National Key Research and Development Program of China Grant 2016YFD0100103, Crop Germplasm Conservation and Utilization Program Grant 2017NWB036-12 from the Ministry of Agriculture of China, and the Agricultural Science and Technology Innovation Program from the Chinese Academy of Agricultural Sciences.

C. Duan and F. Song contributed equally to this work.

The author(s) declare no conflict of interest.

(C) 2019 The American Phytopathological Society studies have shown that the inheritance of maize stalk rot resistance depends on quantitative and qualitative traits (Cao et al. 1996; Chen and Song 1999; Kappelman and Thompson 1966). In stalk rot caused by Gibberella zeae and Colletotrichum graminicola, resistance is mainly associated with quantitative traits, with both additive and dominance effects jointly playing a role (Pe et al. 1993; Yang et al. 2001). Several Fusarium stalk rot resistance loci were discovered and mapped, including the major gene $R f g l$ ( $q R f g l$ ) (Wang et al. 2017; Yang et al. 2004, 2010; Ye et al. 2013) and the minor resistance genes $q R f g 2$ (Zhang et al. 2012), $q R f g 3$ (Ma et al. 2017), and Rgsr8.1 (Chen et al. 2017). In addition, the anthracnose stalk rot resistance locus Rcgl was also mapped (Frey et al. 2011). However, resistance to Pythium stalk rot has been mainly associated with qualitative traits (Cao et al. 1996; Chen and Song 1999; Yang et al. 2005). Only a few Pythium stalk rot resistance genes have been mapped and formally named. These genes include the Pythium stalk rot resistance gene, Rpil (Yang et al. 2005), RpiQI319-1, and RpiQI319-2 (Song et al. 2015). The molecular markers linked with Pythium stalk rot resistance genes could assist in selective breeding for resistance to Pythium stalk rot.

Because of the coevolution of host and pathogen, many resistance genes do not provide durable resistance, with many superior hybrid varieties losing resistance after a few years of large-scale commercial cultivation. Therefore, a continuous discovery of new Pythium stalk rot resistance genes is required to protect maize from stalk rot and minimize yield losses. Simple sequence repeat (SSR) markers, which have advantages such as high reproducibility and codominance, have wide applications in resistance gene mapping. Bulked segregation analysis (BSA) is commonly used to rapidly screen molecular markers that are tightly linked to resistance genes (Michelmore et al. 1991). Maize inbred line X178 exhibits a stable resistance to Pythium stalk rot. Genetic analysis has shown that two independently inherited dominant genes carried by X178 are responsible for the resistance relative to the susceptible parent Ye107. The aim of this study was to finely map stalk rot resistance genes in X178, obtain tightly linked molecular markers, and then, perform allelism testing of adjacent genes. The results of this study 
provide new molecular markers and genetic resources to aid in the breeding of stalk rot-resistant varieties.

\section{MATERIALS AND METHODS}

Plant materials and inoculum. The maize inbred line X178 is highly resistant to $P$. inflatum, which belongs to the PB subgroup (Group B in modern American hybrid) and evolved from the American hybrid P78599 based on genealogy. Resistant X178 and susceptible Ye107 were used as contrasting parents, and they were crossed to generate genetic populations. The derived $90 \mathrm{~F}_{1}$ individuals, $736 \mathrm{~F}_{2}$ individuals, and $690 \mathrm{~F}_{2: 3}$ lines from a cross of $\mathrm{X} 178 \times$ Ye107 were used for phenotypic evaluation and genetic analysis for resistance to $P$. inflatum in $\mathrm{X} 178$. The $\mathrm{F}_{2}$ reciprocal cross-populations were also generated by crossing the resistant lines X178 $\times 1145$ and X178 $\times$ Qi319 that were used for allelism tests of resistance genes. Maize germplasm was preserved in the China National Genebank at the Institute of Crop Sciences, Chinese Academy of Agricultural Sciences.

The Pythium stalk rot pathogen $P$. inflatum isolate P85-67 was used as inoculum in this study, and it was isolated, purified, and stored in the laboratory of the Institute of Crop Sciences, Chinese Academy of Agricultural Sciences.

Inoculation and phenotypic evaluation. The $F_{1}, F_{2}$, and $F_{2: 3}$ populations derived from the same cross between Ye107 and X178 were used to investigate the inheritance of resistance to stalk rot by phenotypic evaluation inoculated with isolate P85-67. All materials were planted at the Changping Experimental Field, Institute of Crop Sciences, Chinese Academy of Agricultural Sciences. The two contrasting parents were planted in the same test field and used as controls.

$P$. inflatum isolate P85-67 was cultured in maize kernels. The inoculum cultivation procedure was according to the procedure in our previous study (Song et al. 2015). First, maize kernels were soaked in tap water overnight for full expansion, and then, the treated maize kernels were cooked in boiling water for $0.5 \mathrm{~h}$. Second, the cooked kernels were put into a triangular flask and autoclaved at $121^{\circ} \mathrm{C}$ for $1 \mathrm{~h}$, after which they were inoculated with $P$. inflatum. Third, the inoculated kernels were incubated in a culture room at $25^{\circ} \mathrm{C}$ with a 0 -h photoperiod for 14 days. Fourth, $50 \mathrm{~g}$ of maize kernels with $P$. inflatum were embedded into 5-cm-deep soil around each plant at the 10-leaf stage of maize.

The plants of parents, $\mathrm{F}_{1}, \mathrm{~F}_{2}$, and $\mathrm{F}_{2: 3}$ populations were investigated for phenotypic evaluation three times at weekly intervals. The typical stalk rot symptoms include browning in lower internodes, spongy stem, wilting, lodging, and plant death at the waxy ripe stage. Thus, the stalks of each plant were longitudinally split to observe the severity of stalk rot based on the degree of discoloration in the internodes. The investigation of stalk rot was completed according to published protocols (Song et al. 2015; Yang et al. 2010). The disease severity was scored using a scale from 1 to 6 , where a score of 1 indicated no symptom appearance on plants at last scoring, a score of 2 indicated no symptoms at the first two scorings and a few symptoms at the last scoring, a score of 3 indicated no symptoms at the second scoring or some symptoms detected at the last scoring, a score of 4 indicated a few visible symptoms observed at the second scoring and symptoms detected at the last scoring, a score of 5 indicated visible symptoms observed at the second scoring, and a score of 6 indicated severe symptoms observed at the first scoring. The plants with scores of 1,2 , or 3 were classified as highly resistant, resistant, and intermediately resistant, respectively; those with scores of 4,5 , or 6 were classified as intermediately susceptible, susceptible, and highly susceptible, respectively.

DNA extraction and pooling for BSA. Genomic DNA was extracted from healthy young leaves according to a previous description (McCouch et al. 1988). The equal amounts of DNA from 10 homozygous resistant and 10 homozygous susceptible $\mathrm{F}_{2}$ plants were mixed to produce resistant and susceptible DNA bulks for BSA (in which equal amounts of DNA from 10 homozygous resistant and 10 homozygous susceptible $\mathrm{F}_{2}$ plants were separately mixed to produce the resistant and susceptible DNA pools), respectively (Michelmore et al. 1991). DNA bulks were prepared by pooling $1 \mu \mathrm{g}$ of DNA of each of the selected plants of the $F_{2}$ population.

Molecular marker analysis and genetic mapping. A total of 810 SSR markers were selected from the MaizeGDB (https://www. maizegdb.org/), and they were used to detect the polymorphism between contrasting parents and two bulks. The BSA polymorphic SSR markers were further tested for the entire $\mathrm{F}_{2}$ mapping population. The polymerase chain reactions (PCRs) were performed on a GeneAmp PCR System 9700 thermal cycler (ABI, Norwalk, CT) in 20- $\mu$ l reaction volumes containing $50 \mathrm{ng}$ of genomic DNA, $0.2 \mu \mathrm{M}$ primer mixture, $1.5 \mathrm{U}$ of Taq DNA polymerase, $0.2 \mathrm{mM}$ deoxy-ribonucleoside triphosphate (dNTPs), and $2.0 \mu \mathrm{l}$ of $10 \times$ buffer. Reactions were performed using the PCR program as follows: $94^{\circ} \mathrm{C}$ for 4 min followed by 35 cycles of $94^{\circ} \mathrm{C}$ for $1 \mathrm{~min}, \sim 50$ to $60^{\circ} \mathrm{C}$ for $1 \mathrm{~min}$, and $72^{\circ} \mathrm{C}$ for $1 \mathrm{~min}$ and a final extension at $72^{\circ} \mathrm{C}$ for $10 \mathrm{~min}$. The PCR products were separated on an $8 \%$ polyacrylamide gel by electrophoresis and stained with a silver nitrate solution for visualization. To finely map the resistant gene, new SSR markers distributed in the target region were designed based on data in MaizeGDB (https://www.maizegdb.org/) with Primer Premier 5.0 (Premier Biosoft International, Palo Alto, CA).

Data analysis and genetic linkage map construction. The segregation patterns of phenotypes and polymorphic SSR markers tested in the genetic mapping population were tested for goodness of fit to Mendelian segregation ratios with chi-squared $\left(\chi^{2}\right)$ analysis. Linkage analysis between molecular markers and the resistance gene was performed using Mapmaker/Exp version 3.0b software with the logarithum of the odds (LOD) score threshold of 3.0 (Lincoln et al. 1992). The Kosambi mapping function was used to estimate the map genetic distances (Kosambi 1943). The maize genome database (https://www.maizegdb.org/) was used as a reference for the physical mapping of resistance genes to chromosomes. A genetic linkage map was constructed using MapDraw in Microsoft Excel macro.

\section{RESULTS}

Genetic analysis of Pythium stalk rot resistance in $\mathrm{X} 178$. The parental population (Ye107 and X178) and $F_{1}, F_{2}$, and $F_{2: 3}$ progeny were inoculated with $P$. inflatum (P85-67) and examined. The results showed that all 60 X178 individuals were highly resistant (Fig. 1A), that all 60 Ye107 individuals were highly susceptible (Fig. 1B), and that all $90 \mathrm{~F}_{1}$ hybrid progeny were disease resistant (Table 1). When examining the $736 \mathrm{~F}_{2}$ progeny, 689 of the individuals were resistant, and 47 were susceptible, fitting to a ratio of 15:1 ( $\chi^{2}=0.0232$, degree of freedom $\left.(\mathrm{df})=1, P=0.8790\right)$. The $690 \quad F_{2: 3}$ families were identified and classified into 297 homozygous resistant, 348 segregating, and 45 homozygous susceptible types, fitting to a ratio of 7:8:1 $\left(\chi^{2}=0.1863\right.$, df $=2$, $P=0.9110$ ) (Table 1). These results suggested that two independently inherited dominant genes were responsible for the resistance to $P$. inflatum in the inbred line X178. The two genes were tentatively designated as RpiX178-1 and RpiX178-2.

Molecular mapping of the stalk rot resistance genes in X178. A total of 810 SSR markers were used for polymorphism analysis between two parents and $F_{1}$ hybrids. The results showed that molecular markers umc0041, umc2047, bnlg1671, umc1744, umc1847, bnlg1444, and umc1313 all exhibited polymorphism between the parents and susceptible and resistant pools (10 extremely resistant and susceptible individuals in each pool, respectively). These seven molecular markers distributed on maize chromosomes 1 and 4 . Therefore, the Pythium stalk rot resistance genes in X178 were likely to be located on chromosomes 1 and 4 . These seven pairs of primers were then used for testing in $690 \mathrm{~F}_{2}$ individuals with explicit phenotype according to their corresponding $\mathrm{F}_{2: 3}$ lines phenotype. 
Linkage analysis between these molecular markers and the resistance genes was performed using Mapmaker/Exp version 3.0b, and it showed that markers umc0041, umc2047, bnlg1671, and umc1744 are linked to the RpiX178-1 gene flanked by markers umc2047 and bnlg1671, with genetic distances of 2.1 and $6.9 \mathrm{cM}$, respectively (Fig. 2A). The molecular markers umc1847, bnlg1444, and umc1313 were linked to the RpiX178-2 gene flanked by markers bnlg1444 and umc1313, with genetic distances of 0.9 and $5.4 \mathrm{cM}$, respectively (Fig. 2B).

To finely map the gene RpiX178-1, all SSR and InDel polymorphism (IDP) markers distributed on maize bin 1.09 to 1.10 were amplified between the parents and the resistant and susceptible pools, indicating that the molecular marker IDP2347 exhibits polymorphism between the resistant and susceptible parents and the resistant and susceptible pools. This marker was then tested in $690 \mathrm{~F}_{2}$ individuals. Linkage analysis showed that IDP2347 was linked to the RpiX178-1 gene, and hence, this gene was mapped between the molecular markers umc2047 and IDP2347 at genetic distances of 2.1 and $1.1 \mathrm{cM}$, respectively. To further narrow the location of the RpiX178-1 gene, the B73 sequence was used as a reference to design SSR molecular markers. Polymorphic analysis was further tested against both parents and the DNA bulks, and then, the polymorphic markers SSRZ8 were found. The individual testing in $690 \mathrm{~F}_{2}$ plants and linkage analysis indicated that marker SSRZ33 was at a genetic distance of $0.6 \mathrm{cM}$ from the RpiX178-1 gene on one side. In summary, the RpiX178-1 gene was finely mapped between the molecular markers SSRZ8 and IDP2347, and it was refined into an $\sim 700-\mathrm{kb}$ interval flanked by these two markers (Fig. 2 and Table 2).

The same approach was used to map RpiX178-2. The molecular markers umc2041 and IDP188 were found to be polymorphic between the resistant and susceptible parents and the resistant and susceptible pools. The two markers were then used for testing in 690
$\mathrm{F}_{2}$ individuals. Linkage analysis showed that umc2041 and IDP188 were both linked to the RpiX178-2 gene, with genetic distances of 1.5 and $2.2 \mathrm{cM}$, respectively, from the RpiX178-2 gene on one side. Finally, the RpiX178-2 gene was further located on chromosome 4 flanked by the markers bnlg1444 and umc2041, with genetic distances of 0.9 and $1.5 \mathrm{cM}$, respectively (Fig. 2 and Table 2). The amplicons for the SSRZ8, IDP2347, and bnlg1444 markers in representative Ye107 and X178 individuals are shown in Figure 3.

Relationships validated by allelism testing. This study shows that the RpiX178-1 and RpiX178-2 genes were mapped to chromosome 1 (bin 1.09) and chromosome 4 (bin 4.08). In a

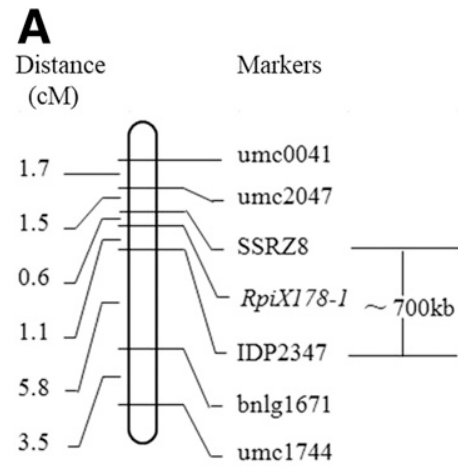

Chromosome 1
B Distance $\quad$ Markers
$(\mathrm{cM})$

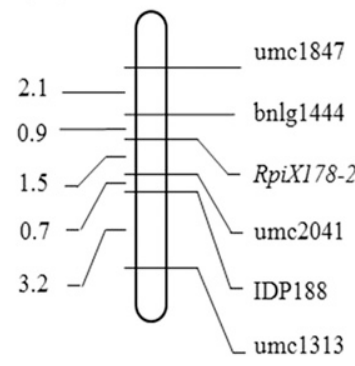

Chromosome 4
Fig. 2. Genetic linkage map of resistance genes A, RpiX178-1 and B, RpiX1782. A, Local linkage map of the RpiX178-1 region on chromosome 1 using reported markers. B, Fine-scale mapping of the RpiX178-2 region on chromosome 4. Loci names are shown on the right side of the map. Genetic distances on the left side of each map are in centimorgans.
A

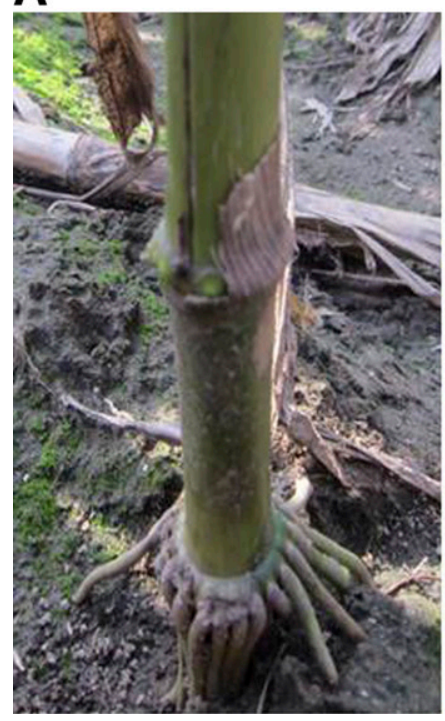

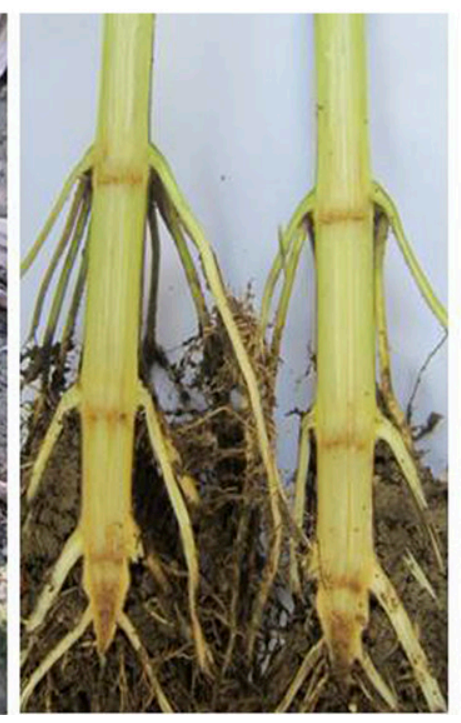

B

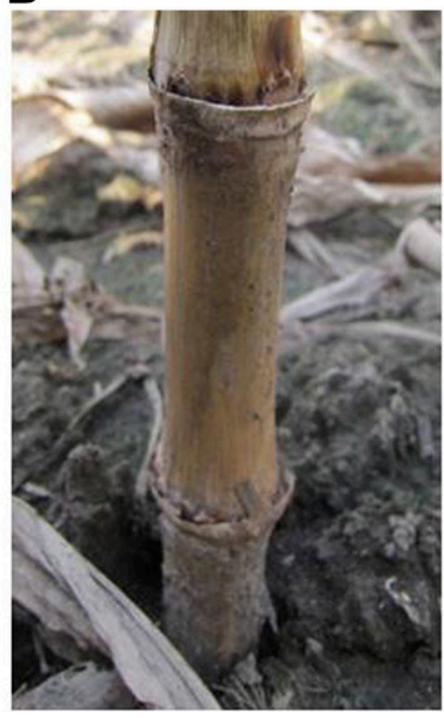

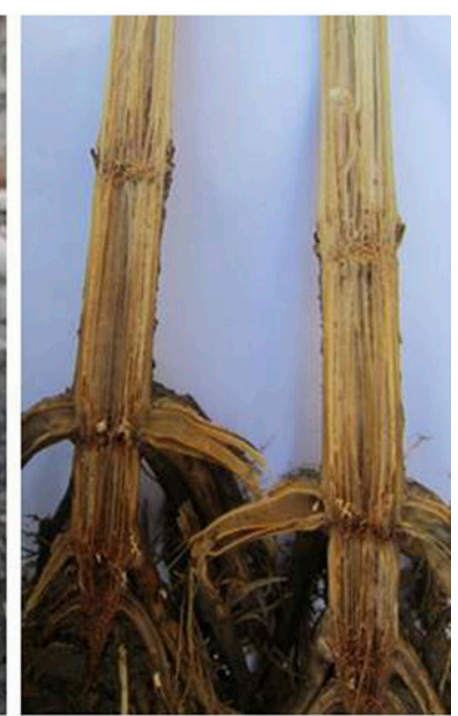

Fig. 1. Field performance of maize inbred lines A, X178 (healthy plants) and B, Ye107 (diseased plants).

TABLE 1. Segregation ratios of the parents, $F_{1}$ and $F_{2}$ individuals, and $F_{2: 3}$ families derived from a cross of Ye107 $\times X 178^{a}$

\begin{tabular}{|c|c|c|c|c|c|c|c|c|}
\hline \multirow[b]{2}{*}{ Parents and crosses } & \multirow[b]{2}{*}{ Generation } & \multirow{2}{*}{$\begin{array}{c}\text { Total number } \\
\text { of plants/families }\end{array}$} & \multicolumn{3}{|c|}{ Observed ratio of $\mathrm{R}: \mathrm{S}$} & \multirow[b]{2}{*}{ Expected ratio } & \multirow[b]{2}{*}{$\chi^{2}$} & \multirow[b]{2}{*}{$P$ value } \\
\hline & & & $\mathrm{R}$ & Seg & $\mathrm{S}$ & & & \\
\hline $\mathrm{X} 178$ & $\mathrm{P}_{\mathrm{R}}$ & 60 & 60 & & 0 & & & \\
\hline Ye107 & $\mathrm{P}_{\mathrm{S}}$ & 60 & 0 & & 60 & & & \\
\hline Ye107 × X178 & $\mathrm{F}_{1}$ & 90 & 90 & & 0 & & & \\
\hline Ye107 × X178 & $\mathrm{F}_{2}$ & 736 & 689 & & 47 & $15: 1$ & 0.0232 & 0.8790 \\
\hline Ye107 × X178 & $\mathrm{F}_{2: 3}$ & 690 & 297 & 348 & 45 & $7: 8: 1$ & 0.1863 & 0.9110 \\
\hline
\end{tabular}

${ }^{a} \mathrm{R}$, highly resistant and resistant; $\mathrm{S}$, susceptible; Seg, segregating. 
TABLE 2. Characteristics and sequences of the five markers linked to the two resistance genes in this study

\begin{tabular}{|c|c|c|c|}
\hline Marker & Primer sequence & Annealing temperature & Linked resistance genes \\
\hline umc2047 & Forward GACAGACATTCCTCGCTACCTGAT & 55 & RpiX178-1 \\
\hline umc2047 & Reverse CTGCTAGCTACCAAACATTCCGAT & & \\
\hline IDP2347 & Forward ACCTTGCACTGCATGTTGG & 56 & RpiX178-1 \\
\hline IDP2347 & Reverse AGTCTTCGAAATTTACCGGG & & \\
\hline bnlg1444 & Forward AGACGACGAAGCTTTTGCAT & 56 & RpiX178-2 \\
\hline bnlg1444 & Reverse GCATGGATGGAGAAAGAGGA & & \\
\hline umc2041 & Forward CTACACAAGCATAGAGGCCTGGAG & 58 & RpiX178-2 \\
\hline umc2041 & Reverse CAGTACGAGACGATGGAGGACAT & & \\
\hline SSRZ8 ${ }^{\mathrm{a}}$ & Forward GCCGTTCTAATTCCCATC & 55 & RpiX178-1 \\
\hline SSRZ8a & Reverse GTTCGCCAGTATGTCTCG & & \\
\hline
\end{tabular}

a Marker developed in this study.

\section{$\begin{array}{llllllllllllllllllllllll}\text { A } & \mathrm{M} & \mathrm{P}_{1} & \mathrm{P}_{2} & \mathrm{~B}_{\mathrm{R}} & \mathrm{B}_{\mathrm{S}} & \mathrm{Rr} & \mathrm{Rr} & \mathrm{Rr} & \mathrm{Rr} & \mathrm{RR} & \mathrm{RR} & \mathrm{RR} & \mathrm{RR} & \mathrm{rr} & \mathrm{rr} & \mathrm{rr} & \mathrm{rr}\end{array}$ $200 \mathrm{bp}$}

$100 \mathrm{bp}$

B

\section{$\begin{array}{llllllllllllllllllll}M & \mathrm{P}_{1} & \mathrm{P}_{2} & \mathrm{~B}_{\mathrm{R}} & \mathrm{B}_{\mathrm{S}} & \mathrm{Rr} & \mathrm{Rr} & \mathrm{Rr} & \mathrm{Rr} & \mathrm{RR} & \mathrm{RR} & \mathrm{RR} & \mathrm{RR} & \mathrm{rr} & \mathrm{rr} & \mathrm{rr} & \mathrm{rr}\end{array}$}

$300 \mathrm{bp}$

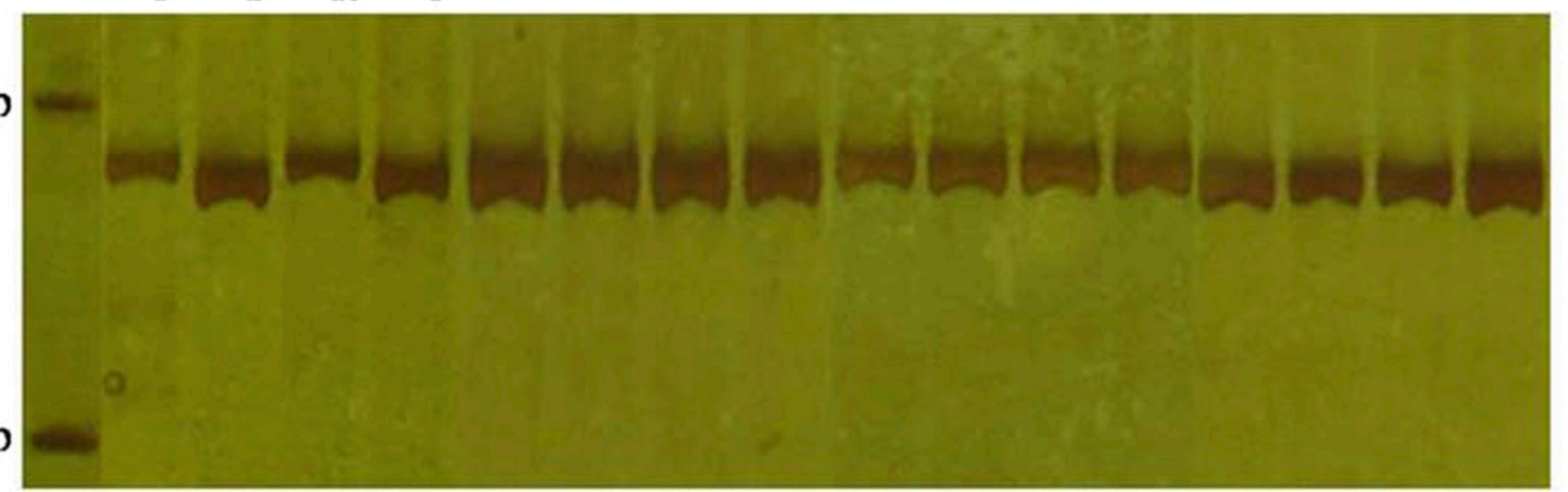

C

$\begin{array}{lllllllllllllllllll}M & \mathrm{P}_{1} & \mathrm{P}_{2} & \mathrm{~B}_{\mathrm{R}} & \mathrm{B}_{\mathrm{S}} & \mathrm{Rr} & \mathrm{Rr} & \mathrm{Rr} & \mathrm{Rr} & \mathrm{RR} & \mathrm{RR} & \mathrm{RR} & \mathrm{RR} & \mathrm{rr} & \mathrm{rr} & \mathrm{rr} & \mathrm{rr}\end{array}$

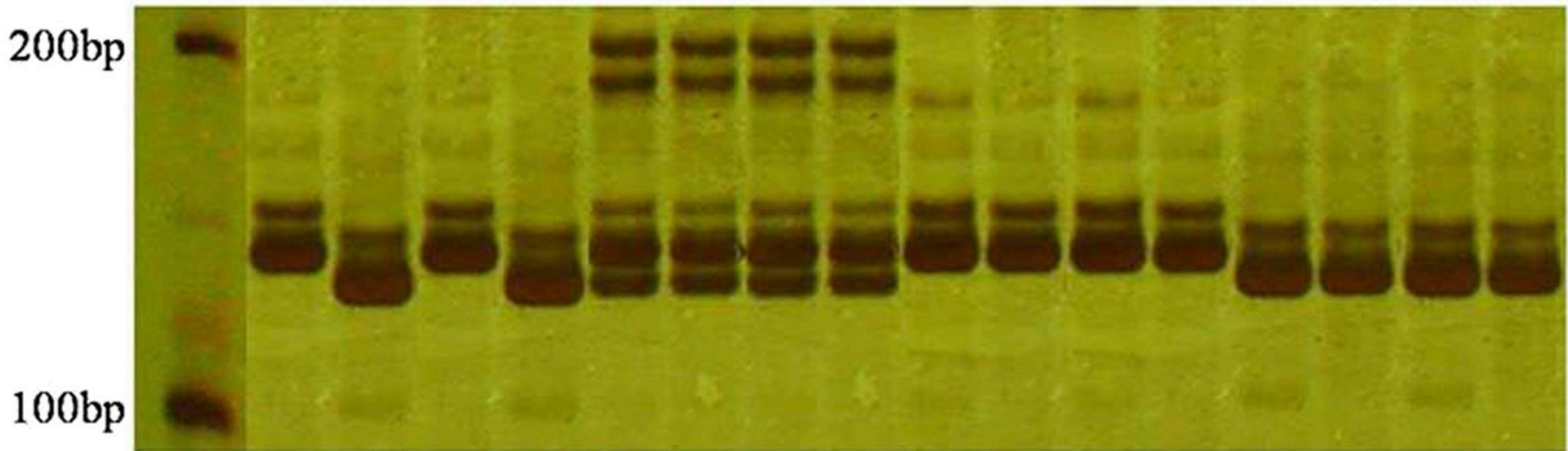

Fig. 3. Amplification patterns of molecular markers A, SSRZ8, B, IDP2347, and C, bnlg1444 linked with resistance genes in this study. $B_{R}$, resistant bulk; $B_{S}$, susceptible bulk; M, 100-bp DNA ladder; $\mathrm{P}_{1}, \mathrm{X} 178 ; \mathrm{P}_{2}$, Ye107; RR, homozygous resistant plants; Rr, heterozygous resistant plants; and rr, homozygous susceptible plants. 
previous study, the resistant inbred line 1145, susceptible Y331, and their $\mathrm{F}_{2}$ and $\mathrm{BC}_{1} \mathrm{~F}_{1}$ progenies were inoculated with $P$. inflatum, and the resistance gene Rpil was mapped between the molecular markers bnlg1937 and agrr286OPA-0 $4_{880}$ on chromosome 1 . In another study, the gene RpiQI319-1 carried by inbred line Qi319 with resistance to Pythium stalk rot was mapped between the molecular markers SSRZ33 and SSRZ47 on chromosome 1. In addition, using the $\mathrm{BC}_{8} \mathrm{~F}_{1}$ population derived from 1145 and $\mathrm{Y} 331$, the minor gene $q R f g 2$ resistance to Fusarium stalk rot was finely mapped to a $300-\mathrm{kb}$ region on chromosome 1 flanked by CAPSZ459 and SSRZ319 markers. To verify the relationship between the previous stalk rot resistance genes $q R f g 2, R p i Q I 319-1$, and Rpil and the genes RpiX178-1 and RpiX178-2, the inbred maize lines X178, 1145, and Qi-319 were used to construct hybrids. The progeny populations were inoculated with $P$. inflatum, and disease resistance was examined using allelism testing. The resistance determination results showed that all three inbred lines exhibited resistance to Pythium stalk rot. Moreover, the $F_{1}$ progeny of the reciprocal crosses between X178 $\times$ Qi319 and X178 $\times 1145$ were all disease resistant, whereas the $\mathrm{F}_{2}$ progeny from the reciprocal crosses had some susceptible individuals (Table 3). Therefore, the X178 resistance genes differ from the resistance genes in the 1145 and Qi319 lines. These findings confirm that the two genes, RpiX178-1 and RpiX178-2, are not identical to previously identified Pythium stalk rot resistance genes. Thus, they are new Pythium stalk rot resistance genes.

\section{DISCUSSION}

Extensive monoculturing will often result in a decrease or loss of resistance in the cultivated variety and create a conducive environment for plant pathogens to invade and proliferate. Under artificial selection conditions, resistant resources can be selectively bred to obtain disease-resistant varieties and prevent pathogen invasion. In maize, Pythium stalk rot, which is caused by $P$. inflatum, is an important pathogen with a dearth of research focused on resistance. Resistance identification studies have verified that the inbred maize line X178 exhibits a high to moderate resistance to multiple diseases, such as Southern corn leaf blight, Southern rust, Fusarium ear rot, and Pythium stalk rot (Duan et al. 2015a, 2015b). Currently, little has been reported pertaining to Pythium stalk rot resistance genes in inbred lines. Herein, the X178 line was inoculated with $P$. inflatum under artificial conditions; then, resistance inheritance was performed, and resistance genes were mapped in X178.

When conducting a resistance evaluation, the Ye107 line (susceptible) with no inoculation was utilized as a blank control, and the determination was carried out during the adult stage. The control samples did not show any signs of Pythium stalk rot, thus showing that the experimental field did not contain any Pythium stalk rot contamination. In the inoculated groups, individuals that exhibited a susceptibility to Pythium stalk rot were examined microscopically during the early stage of disease. The examination showed the presence of $P$. inflatum in the pith of the base stem, thus confirming that the inoculation can effectively induce stalk rot, which is consistent with previous studies (Yang et al. 2005). Within the $\mathrm{F}_{2}$ progeny, the resistance determination identified 689 moderately resistant and 47

TABLE 3. The phenotypes of $F_{1}$ and $F_{2}$ progenies of the reciprocal crosses between resistant inbred lines X178, 1145, and Qi319 used in allelism testing

\begin{tabular}{lcccc}
\hline Hybrids & Generation & $\begin{array}{c}\text { Total number } \\
\text { of individuals }\end{array}$ & Resistant & Susceptible \\
\hline X178 × 1145 & $\mathrm{F}_{1}$ & 50 & 50 & 0 \\
X178 × 1145 & $\mathrm{F}_{2}$ & 458 & 409 & 49 \\
X178 $\times$ Qi319 & $\mathrm{F}_{1}$ & 55 & 55 & 0 \\
X178 $\times$ Qi319 & $\mathrm{F}_{2}$ & 416 & 373 & 43 \\
\hline
\end{tabular}

susceptible individuals. The segregation ratio of 15:1 suggested two dominant genes in X178. In $690 \mathrm{~F}_{2: 3}$ families, a segregation ratio of 7:8:1 further confirmed that X178 Pythium stalk rot resistance is controlled by two independent dominant genes denoted RpiX178-1 and RpiX178-2. The RpiX178-1 gene was mapped to a $700-\mathrm{kb}$ physical interval on chromosome 1 . The RpiX178-2 gene was mapped on chromosome 4 (bin 4.08), located between markers bnlg1444 and umc2041, with the genetic interval of $2.4 \mathrm{cM}$.

Maize stalk rot is a relatively complex disease that is affected by multiple factors, such as the pathogen type, maize genetic background, and environment. In one study, Fusarium resistance in the 1145 inbred line was found to be controlled by the dominant gene $R f g 1$, which was mapped to chromosome 6 (Yang et al. 2004). However, in the same line, $P$. inflatum resistance is controlled by Rpil, a dominant gene on chromosome 4 (Yang et al. 2005). These findings suggest that different pathogens may elicit a different stalk rot resistance even within the same maize line. Herein, the two independent dominant genes, RpiX178-1 and RpiX178-2, carried by $\mathrm{X} 178$ are responsible for its resistance to $P$. inflatum relative to the susceptible parent Ye107, which is different from the findings in the populations from a cross between $1145 \times \mathrm{Y} 331$, where resistance is controlled by a single gene (Yang et al. 2005).

In this study, the RpiX178-1 gene was found to be tightly linked to the molecular markers SSRZ8 and IDP2347 that are located on chromosome 1. Therefore, these two markers might be used for molecular marker-assisted breeding. Currently, many resistance genes have been found on maize chromosome 1, such as two quantitative trait loci (QTLs) associated with gray leaf spot disease resistance (Maroof et al. 1996) and resistance genes associated with Fusarium ear rot (Maschietto et al. 2017), northern leaf blight (Chung et al. 2011), and Gibberella stalk rot (Zhang et al. 2012). These findings suggest that maize chromosome 1 may be a region where resistance genes are concentrated. In this study, RpiX178-1 and RpiX178-2 were located on chromosomes 1 and 4, whereas in the 1145 line, G. zeae and P. inflatum resistance genes, Rfgl and Rpil, are located on chromosomes 6 and 4, respectively (Yang et al. 2004, 2005). Also in the 1145 line, G. zeae resistance has been associated with the QTLs $q R f g 1$ and $q R f g 2$, which are located on chromosomes 10 and 1, respectively (Yang et al. 2005; Zhang et al. 2012). Herein, four molecular markers linked with $q R f g 2$ and Rpil were utilized to carry out linkage analysis of the Ye107 $\times \mathrm{X} 178 \mathrm{~F}_{2}$ population. The results showed that the four markers were not linked to the RpiX178-1 and RpiX178-2 genes. To further verify the accuracy of the experimental results, X178 and 1145 hybrids were generated, the $\mathrm{F}_{2}$ progenies were inoculated, and resistant individuals were discovered. This finding further confirms that the resistance genes in X178 and 1145 differ and shows that the experiment results are accurate and reliable. Therefore, the resistance genes RpiX178-1 and RpiX178-2 are novel Pythium stalk rot resistance genes that have not been previously reported.

Information about the genetic mechanisms of $P$. inflatum resistance in maize have been limited, which presents a major bottleneck in the development of new maize varieties with Pythium stalk rot resistance. In this study, the resistance to $P$. inflatum in X178 was examined, and two dominant genes (RpiX178-1 and RpiX178-2) associated with Pythium stalk rot resistance were characterized. To our knowledge, this is the first report on the resistance to $P$. inflatum in inbred lines X178. The molecular markers SSRZ8 and bnlg1444 were found to be tightly linked to these two genes and thus, will facilitate breeding new resistant cultivars by marker-assisted selection.

\section{LITERATURE CITED}

Cao, R. H., Wang, F. R., Wang, X. L., Fu, Y. H., Chen, Y. X., Ren, J. H., Wang, Z., and Ma, P. Q. 1996. Study on the maize genetic resistance of maize to Pythium inflatum Matthews. Hereditas 18:4-6. 
Chen, Q., Song, J., Du, W. P., Xu, L. Y., Jiang, Y., Zhang, J., Xiang, X. L., and Yu, G. R. 2017. Identification, mapping, and molecular marker development for Rgsr8.1: A new quantitative trait locus conferring resistance to Gibberella stalk rot in maize (Zea mays L.). Front. Plant Sci. 8:1355.

Chen, S. J., and Song, T. M. 1999. Resistance to maize stalk rot (Pythium inflatum Mattews): Simple genetic control of a pair of genes. J. China Agric. Univ. 1:56.

Chung, C. L., Poland, J., Kump, K., Benson, J., Longfellow, J., Walsh, E., Balint-Kurti, P., and Nelson, R. 2011. Targeted discovery of quantitative trait loci for resistance to northern leaf blight and other diseases of maize. Theor. Appl. Genet. 123:307-326.

Duan, C. X., Jiang, K., Qin, Z. H., Sun, S. L., Song, F. J., and Wang, X. M. 2015a. Identification of resistance marker genotype and genetic diversity of maize germplasm with resistance to southern corn rust. J. Plant Prot. 42: 899-907.

Duan, C. X., Wang, X. M., Wu, X. F., Yang, Z. H., Song, F. J., Zhao, L. P., Sun, S. L., and Zhu, Z. D. 2015b. Analysis of maize accessions resistance to Pythium stalk rot and Fusarium ear rot. J. Plant Genet. Res. 16:947-954.

Duan, C. X., Zhu, Z. D., Wu, X. F., Yang, Z. H., and Wang, X. M. 2012. Screening and evaluation of maize germplasm for resistance to five diseases and Asian corn borer. J. Plant Genet. Res. 13:169-174.

Frey, T. J., Weldekidan, T., Colbert, T., Wolters, P. J. C. C., and Hawk, J. A. 2011. Fitness evaluation of Rcg1, a locus that confers resistance to Colletotrichum graminicola (Ces.) G. W. Wils. using near-isogenic maize hybrids. Crop Sci. 51:1551-1563.

Kappelman, A. J., and Thompson, D. L. 1966. Inheritance of resistance to diplodia stalk-rot in corn. Crop Sci. 6:288-290.

Khokhar, M. K., Hooda, K. S., Sharma, S. S., and Singh, V. 2014. Post flowering stalk rot complex of maize - present status and future prospects. Maydica 59:226-242.

Kosambi, D. D. 1943. The estimation of map distance from recombination values. Ann. Eugen. 12:172-175.

Lincoln, S., Daly, M., and Lander, E. 1992. Constructing Genetic Maps with Mapmaker/EXP30 Whitehead Institute Tech. Rep., 3rd Ed. Whitehead Institute, Cambridge, MA.

Ma, C., Ma, X., Yao, L., Liu, Y., Du, F., Yang, X., and Xu, M. 2017. qRfg3, a novel quantitative resistance locus against Gibberella stalk rot in maize. Theor. Appl. Genet. 130:1723-1734.

Maroof, M. A., Yue, Y. G., Xiang, Z. X., Stromberg, E. L., and Rufener, G. K. 1996. Identification of quantitative trait loci controlling resistance to gray leaf spot disease in maize. Theor. Appl. Genet. 93:539-546.
Maschietto, V., Colombi, C., Pirona, R., Pea, G., Strozzi, F., Marocco, A., Rossini, L., and Lanubile, A. 2017. QTL mapping and candidate genes for resistance to Fusarium ear rot and fumonisin contamination in maize. BMC Plant Biol. 17:20.

Michelmore, R. W., Paran, I., and Kesseli, R. V. 1991. Identification of markers linked to disease-resistance genes by bulked segregant analysis: A rapid method to detect markers in specific genomic regions by using segregating populations. Proc. Natl. Acad. Sci. USA 88: 9828-9832.

Pe, M. E., Gianfranceschi, L., Taramino, G., Tarchini, R., Angelini, P., Dani, M., and Binelli, G. 1993. Mapping quantitative trait loci (QTLs) for resistance to Gibberella zeae infection in maize. Mol. Gen. Genet. 241:11-16.

Song, F. J., Xiao, M. G., Duan, C. X., Li, H. J., Zhu, Z. D., Liu, B. T., Sun, S. L., Wu, X. F., and Wang, X. M. 2015. Two genes conferring resistance to Pythium stalk rot in maize inbred line Qi319. Mol. Genet. Genomics 290: 1543-1549.

Wang, C., Yang, Q., Wang, W., Li, Y., Guo, Y., Zhang, D., Ma, X., Song, W., Zhao, J., and Xu, M. 2017. A transposon-directed epigenetic change in ZmCCT underlies quantitative resistance to Gibberella stalk rot in maize. New Phytol. 215:1503-1515.

Yang, D. E., Chen, S. J., Wang, Y. G., Zhang, C. L., Li, S. R., and Wang, B. 2001. Genetic analysis of maize stalk rot (Pythium inflatum Mattews) resistance gene. Acta Phytopathol. Sin. 31:315-318.

Yang, D. E., Jin, D. M., Wang, B., Zhang, D. S., Nguyen, H. T., Zhang, C. L., and Chen, S. J. 2005. Characterization and mapping of Rpil, a gene that confers dominant resistance to stalk rot in maize. Mol. Genet. Genomics 274:229-234.

Yang, D. E., Zhang, C. L., Zhang, D. S., Jin, D. M., Weng, M. L., Chen, S. J., Nguyen, H., and Wang, B. 2004. Genetic analysis and molecular mapping of maize (Zea mays L.) stalk rot resistant gene Rfgl. Theor. Appl. Genet. 108:706-711.

Yang, Q., Yin, G., Guo, Y., Zhang, D., Chen, S., and Xu, M. 2010. A major QTL for resistance to Gibberella stalk rot in maize. Theor. Appl. Genet. 121:673-687.

Ye, J., Guo, Y., Zhang, D., Zhang, N., Wang, C., and Xu, M. 2013. Cytological and molecular characterization of quantitative trait locus $q R f g 1$, which confers resistance to Gibberella stalk rot in maize. Mol. Plant-Microbe Interact. 26:1417-1428.

Zhang, D., Liu, Y., Guo, Y., Yang, Q., Ye, J., Chen, S., and Xu, M. 2012. Finemapping of $q R f g 2$, a QTL for resistance to Gibberella stalk rot in maize. Theor. Appl. Genet. 124:585-596. 